\title{
A Machine Vision Quality Control System for Industrial Acrylic Fibre Production
}

\author{
Paulo Heleno \\ INETI-DOP, Estrada do Paço do Lumiar, 22, 1649-038 Lisbon, Portugal \\ Email: pheleno@dop.ineti.pt \\ Roger Davies \\ INETI-DOP, Estrada do Paço do Lumiar, 22, 1649-038 Lisbon, Portugal \\ Email: rdavies@dop.ineti.pt

\section{Bento A. Brázio Correia} \\ INETI-DOP, Estrada do Paço do Lumiar, 22, 1649-038 Lisbon, Portugal \\ Email: bcorreia@dop.ineti.pt \\ João Dinis \\ INETI-DOP, Estrada do Paço do Lumiar, 22, 1649-038 Lisbon, Portugal \\ Email:jdinis@dop.ineti.pt
}

Received 1 August 2001

\begin{abstract}
This paper describes the implementation of INFIBRA, a machine vision system used in the quality control of acrylic fibre production. The system was developed by INETI under a contract with a leading industrial manufacturer of acrylic fibres. It monitors several parameters of the acrylic production process. This paper presents, after a brief overview of the system, a detailed description of the machine vision algorithms developed to perform the inspection tasks unique to this system. Some of the results of online operation are also presented.
\end{abstract}

Keywords and phrases: quality control, machine vision, textile industry.

\section{INTRODUCTION}

Quality control is becoming increasingly more important in today's manufacturing industries. Customers demand cheap but high quality products. For an efficient and successful production, manufacturers must rely on a robust quality control system. These systems can prevent product rejection by detecting all defective products and simultaneously avoid false defect detections. Also, an automated quality control system integrated into the manufacturing process can detect production deviations early and thus avoid waste.

Compared to the traditional quality control tasks performed by humans, automated quality control systems offer various advantages, including the ability to work in hazardous environments 24 hours a day, and in some tasks perform quicker measurements with higher accuracy and consistency than humans. Additionally, when the automated quality control system is computer-controlled, the results of monitoring can easily be integrated into the manufacturer's statistical process control.
Machine vision quality control systems play a growing role in modern manufacturing quality control systems. One of the reasons explaining this growth is that quick, accurate noncontact measurement and complex feature analysis can now be performed by low-cost machine vision systems.

\section{PROBLEM DESCRIPTION}

The production of acrylic fibres on the manufacturer's production lines begins with the extrusion of a liquid polymer through spinnerets (thin metal diaphragms perforated by thousands of small holes) into a high-temperature water bath. The solidifying fibres form six parallel, flattened bundles, or tapes, each approximately of $110 \mathrm{~mm}$ wide and spaced approximately $15 \mathrm{~mm}$ apart. The tapes are drawn through the production line machinery where they are progressively stretched and dried over a series of heated metal cylinders.

The width of each tape and the interval between them has an important influence on the quality of the fibres produced 


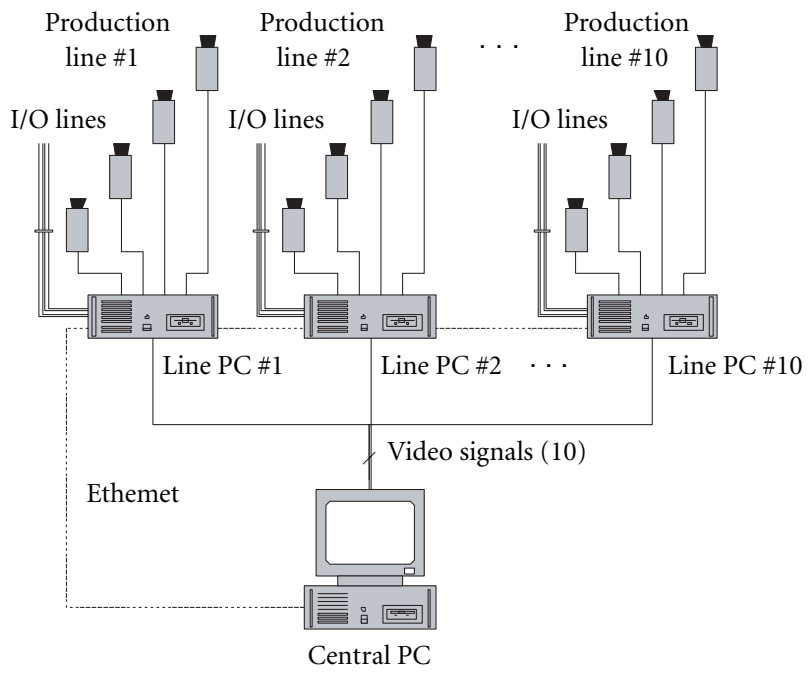

FIGURE 1: System hardware configuration.

and so it is essential that these parameters be kept within strict tolerances. In addition, it is not uncommon for fibres to break and become wrapped around the cylinders. If such a "wrap" remains undetected for more than a few minutes it may be necessary to stop the production line. In such cases it can take up to 24 hours to recommence production.

The manufacturer in question has ten production lines in continuous operation, each approximately $40 \mathrm{~m}$ in length. Before the introduction of the INFIBRA system, a team of operators performed periodic, manual visual inspection of each line in conditions of very high ambient temperature and near $100 \%$ humidity. The known limitations of manual inspection, aggravated by the adverse ambient conditions, were important factors in the decision to implement an alternative, automated inspection solution that would improve both quality and productivity.

\section{OBJECTIVES}

The overall objective of the INFIBRA system is to improve quality control of acrylic fibre production by automatically and reliably detecting manufacturing anomalies in real time. The specific objectives can be summarized as follows:

(1) Perform continuous, automatic inspection of ten production lines.

(2) Detect anomalies in fibre tape width and spacing.

(3) Detect gaps between the fibres that form each tape.

(4) Detect fibres wrapped around the production line cylinders (wraps).

Permit control and monitoring of all inspection activities from a central control unit.

\section{SYSTEM DESCRIPTION}

The INFIBRA system developed by INETI (Instituto Nacional de Engenharia e Tecnologia Industrial) consists of

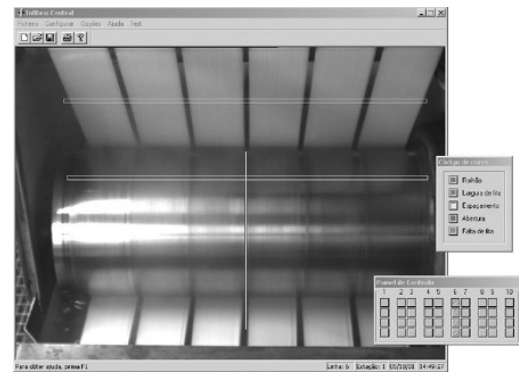

FIGURE 2: System interface.

a network of industrial personal computers (PCs), each equipped with a low-cost frame grabber. Each acrylic fibre production line has a dedicated PC that performs automatic inspection through specially designed metrology algorithms, using four video cameras located at key positions on the production line. The ten PCs communicate with a central control PC via an Ethernet network connection (Figure 1).

The user at the central control room manages the inspection process through an interactive application running in Microsoft Windows (Figure 2). The user can select and view, in real time, the images captured by each of the cameras on the production line.

To guarantee controllability and maintain a reasonable performance, the inspection is limited to small areas of the images, the inspection windows (the narrow horizontal rectangles that can be seen in Figure 2). The user is free to place as many inspection windows as he thinks necessary, and also to choose the best positions to place the inspection windows (areas with stronger contrast, small temporal variation, etc.).

In system configuration mode, before starting inspection, the user can choose, among other options, where to place the inspection windows, enter the limits for each monitored parameter and specify the frequency at which the inspection should be performed. This application also executes a semiautomatic calibration procedure for each of the inspection windows defined.

An extra marker (the narrow vertical bar seen in Figure 2) is available, during the configuration mode, to help detecting any misalignment of the camera relative to the cylinders. When running in automatic inspection the system warns the user at the central station when defects are detected. Defect alarms are classified into two categories, according to their duration. When the system detects a defect, an alarm level 1 is declared and a yellow warning icon appears on the application's control panel. If the defect quickly disappears the warning icon reverts back to green automatically, meaning that the short-duration defect has disappeared of its own accord. If, on the other hand, the alarm persists beyond a configurable period of time, a level 2 alarm is declared. In this case a red icon appears on the control panel and a message is sent to one of the line operator's pagers to go to the inspection station and try to fix the problem. When the problem is fixed, the operator activates a push-button at the inspection station that changes the alarm icon to green. 
When an alarm is detected the system displays the corresponding inspection station camera image to the operator at the central station. The detected defect is overlaid on the image using a color code indicating which class of defect has been detected.

\section{ALGORITHMS}

The algorithms developed for this system include the fibre tape and wrap detection algorithms, the detection criteria, and the calibration algorithms.

\subsection{Fibre tape and wrap detection}

In the images obtained at each inspection station the fibre tapes and wraps appear as almost vertical bands (Figure 3). These bands contrast with the background cylinder, meaning that fibre tape and wrap segmentation is essentially an edge detection and edge matching process. However, neither illumination nor contrast is constant within any inspection window. Illumination conditions also change dramatically throughout a 24 -hours period. A simple approach, such as segmentation by a constant threshold applied to the whole inspection window is therefore impracticable. The approach described here, using the local maxima of the horizontal image gradient, is based on local properties and attempts to attenuate the effect of both spatial and temporal variations within the inspection window.

\subsubsection{Horizontal gradient maxima}

The horizontal gradient is computed by convolution. An image of the horizontal gradient modulus is constructed by convolving a $3 \times 3$ matrix over the original image of the inspection window, see (1). This matrix weights the pixels closest to the centre, giving the advantage of smoothing some of the noise [1]. The resulting maxima are caused not only by the transitions between tape and background, but also by noise and other image features resulting from shadows and oxidation of the cylinders. Properties of the maxima resulting from tape transitions will be exploited to segment them from other maxima.

Matrix used to compute the horizontal gradient

$$
\left[\begin{array}{lll}
-1 & 0 & +1 \\
-2 & 0 & +2 \\
-1 & 0 & +1
\end{array}\right]
$$

The resulting gradient image (Figure $7 \mathrm{~b}$ ) is scanned and local maxima are marked. The image is iterated from left to right, line by line. Every pixel that is brighter than its two nearest neighbors and has a value above a predefined noisefiltering threshold is marked as a local maximum, resulting in a binary image of local maxima. In situations where no single maximum exists, like in the example of Figure $4 \mathrm{~b}$, the central point of the plateau is chosen as the local maxima.

In Figure $7 \mathrm{c}$ not only tape edges are visible, but also

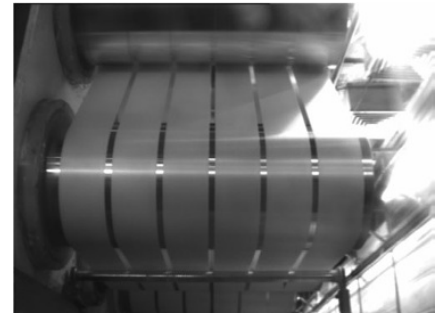

FIgURe 3: Typical image captured at an inspection station showing six fibre tapes and several cylinders.

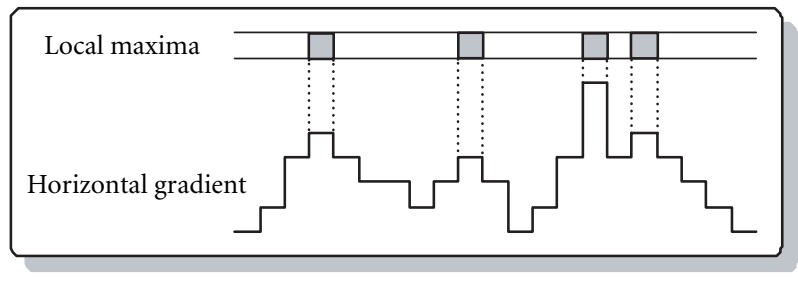

(a)

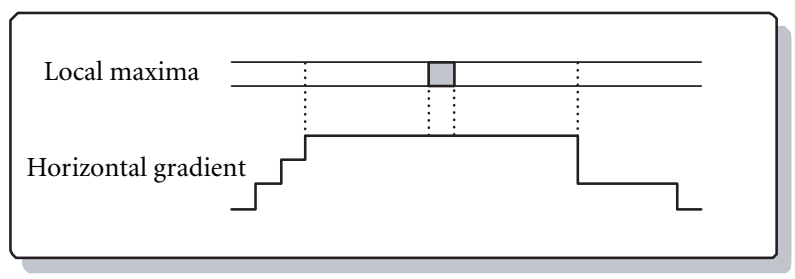

(b)

FIgURE 4: Local maxima detection.

several other maxima caused by shadows, small fibre folds, noise, and even the fibres that form the tape. These other maxima will be eliminated in subsequent steps of the algorithm.

\subsubsection{Edge detection}

Local maxima are then grouped into predominantly vertical segments. A specialized version of the boundary-tracking algorithm described in [1] was adapted for predominantly vertical transitions. The maxima image is analyzed from top to bottom, and for every maximum an iterative search is performed for other maximal neighbors below it. If a single neighbor is found, the position of the previous point is added to a point list and the focus moves to that neighbor. The search continues until no neighbor can be found or until multiple neighbors are found. The reason to stop when multiple choices are detected is because branch selection is simplified using a continuity criterion and this data is only available at the next stage of the algorithm. When the search can progress no further, the resulting 


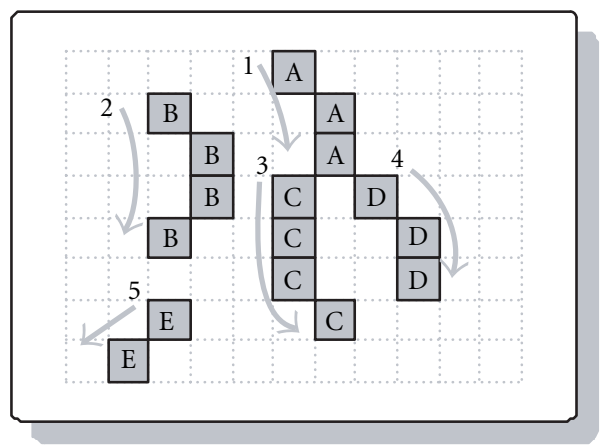

(a)

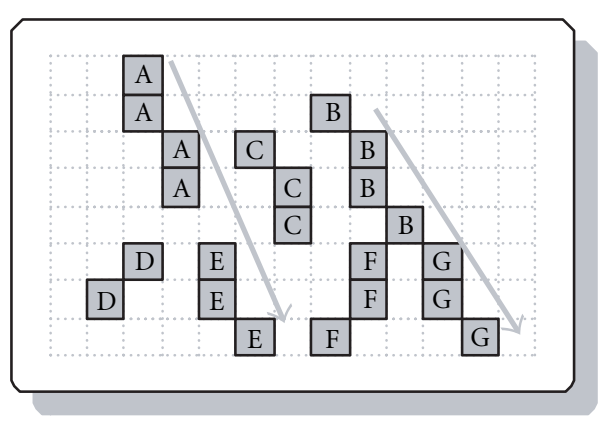

(b)

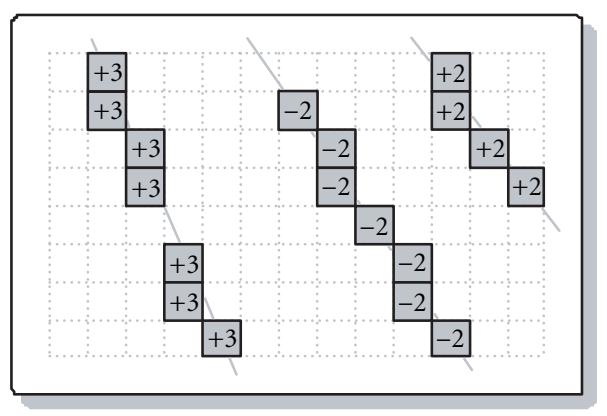

(c)

Figure 5: Edge detection.

point list is stored as a segment in a segment list. The process is repeated until all segments have been identified (Figure 5a).

Segments that can be grouped into longer segments are then merged. Neighboring segments are merged that approximately follow the same direction and are not farther apart than a predefined distance. If more than one neighboring segment is identified, a candidate list is created. The slope and position of each candidate segment is then analyzed and the one that matches the first segment more closely is selected. The process is repeated until no further segment merging can be achieved. In the example shown in Figure $5 b$, segment A will be merged with segment $E$ and segment $B$ will

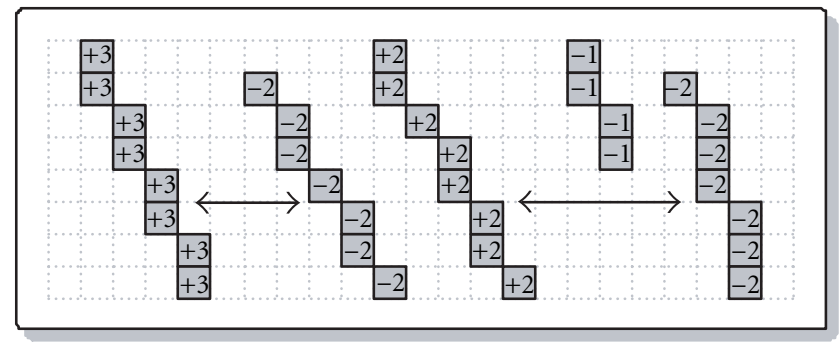

(a)

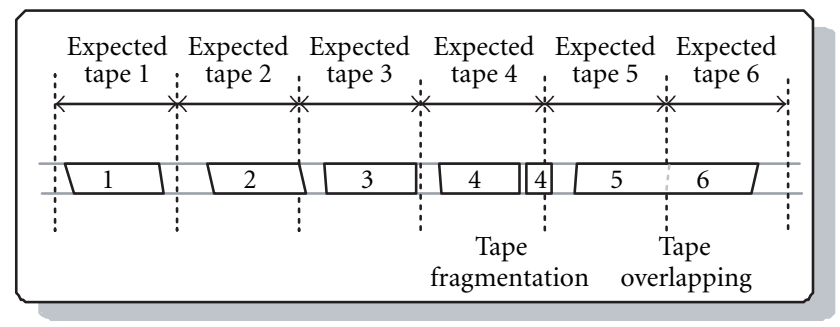

(b)

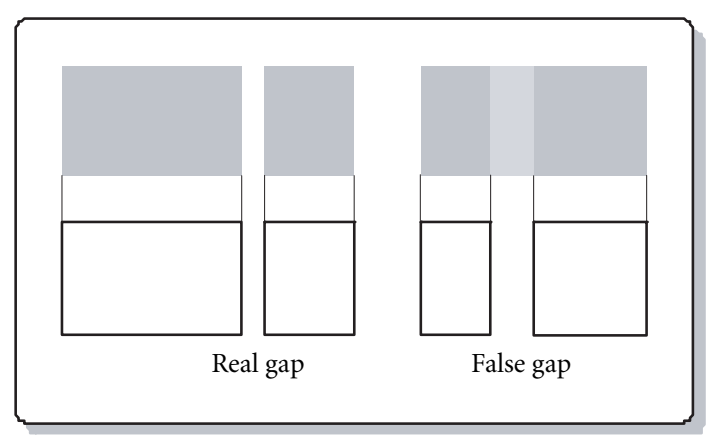

(c)

FIgure 6: Tape identification.

be merged with segment $\mathrm{G}$ (segment $\mathrm{F}$, although near to segment $B$, does not follow its direction as closely as segment $G$ does). No other segments shall be merged because none of the remaining segments meet the proximity and continuity criteria.

Small segments are then eliminated from the segment list. The small segments are kept until this stage because they could be used to complete other segments, however, if they were not merged at this point they have no further use. Segments that are shorter than a third of the total inspection window height are removed as they result mainly from noise in the image and other small nonrelevant features.

Segments are then classified as edges, constructed from one or more segments, and containing information about the level of contrast in the original image. The edges are classified according to the type of the contrast variation they 


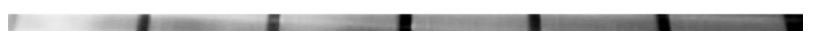

(a)

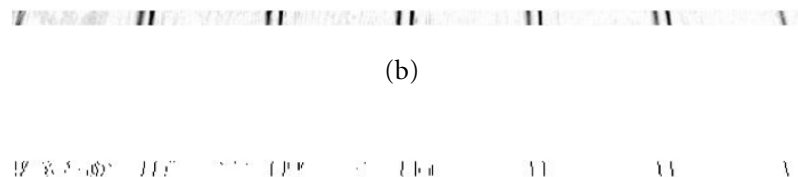

(c)

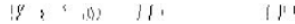

(d)

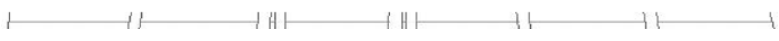

(e)

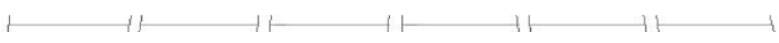

(f)

FIGURE 7: Tape detection steps: (a) original image; (b) negative horizontal gradient; (c) horizontal gradient local maxima; (d) segments; (e) tapes with gaps; (f) false gaps removed.

exhibit, dark-to-light or light-to-dark, shown as the dark and light edges, respectively, in Figure 7d. The level of contrast is defined by the average horizontal contrast at each of the segment points. A linear regression interpolation of the segment is also computed. This representation smoothes small errors that often occur in the detection of the transition and is used to calculate edge coordinates with greater precision (Figure 5c).

\subsubsection{Edge matching and tape identification}

The next step in the algorithm matches pairs of edges to their corresponding tape. The edges must be sorted by their horizontal coordinate. This coordinate is estimated along the mid section of the inspection window using the edge's linear regression representation. Starting from the left, the first dark-to-light edge is picked and matched with the next lightto-dark edge. If two or more adjacent edges of the same type are found then the one with the strongest contrast is selected (Figure 6a). The algorithm described here for the detection of bright tapes over a dark background is also usable for dark tapes over a lighter background. In such a case light-to-dark edges are matched with dark-to-light edges. The information about the type of tape-to-cylinder contrast comes from the calibration procedure described in Section 5.3.

Sometimes the fibres that form a tape separate laterally and form a gap. Such gaps must not be confused with the interval between adjacent tapes. Where gaps exist a tape is effectively broken into fragments, which must be merged to obtain the true width of each tape. In addition to these real gaps, small variations in the fibre brightness or color can cause false gaps (and apparent tape fragmentation) as seen in the two central tapes in Figure 7e. One of the results of the calibration process (described in Section 5.3) is the expected position of each tape. With this information, each fragment can be assigned to the appropriate tape. A fragment is assigned to the calibration tape that is nearest to its central point (Figure 6b).

False gaps are subsequently identified using a brightness criterion, which states that if the average brightness within the gap differs by less than $10 \%$ from the average brightness of the tape then the gap is considered false (Figure 6c). A comparison of Figure $7 \mathrm{e}$ with Figure $7 \mathrm{f}$ shows how two such false gaps have been successfully identified.

Another possible occurrence is tape overlapping, which usually causes the detection of an exceedingly wide tape. A percentage of the detected tape is assigned to each of the expected tapes that lie beneath it. The tape detection algorithm has to deal with this kind of events because, although this overlapping would cause a tape spacing error, it might not cause missing tape error.

\subsection{Detection criteria}

The INFIBRA system monitors tape presence and width, the space between tapes, the size of any gaps within tapes, and finally wrap width (Figure 8). Tape length and the space between tapes both have minimum and maximum limits beyond which the system will signal an alarm. Gap and wrap widths also have maximum limits, however, small gaps and wraps are ignored (Figure 10). The system operator can define these limits for each type or batch of fibre.

The detection of the presence of each tape depends on information produced by the calibration process. A particular tape is declared present if the intersection between that tape and the corresponding tape detected during calibration has an effective length greater than a predefined limit (Figure 9). The effective width is defined as the total width of the tape's individual fragments.

\subsection{Calibration}

The calibration procedure is executed, using an image with known characteristics, each time a new batch of fibre is produced. The calibration results include, for each inspection window, the pixel size, the position and width of each tape and an optimal noise-filtering threshold for the horizontal gradient maxima. The operator must introduce some information manually. In the tape calibration procedure, the operator specifies the number of tapes present in the image, their width in millimeters, and their type (light or dark). The tape calibration algorithm automatically finds an optimal threshold and computes the pixel size. An iterative approach is employed to determine the noise-filtering threshold. The tape detection algorithm is executed successively using different threshold values until an optimal value is found. This algorithm, when executed with the optimal threshold, should return the number of tapes specified by the operator. Starting with a high threshold with which no tapes are 


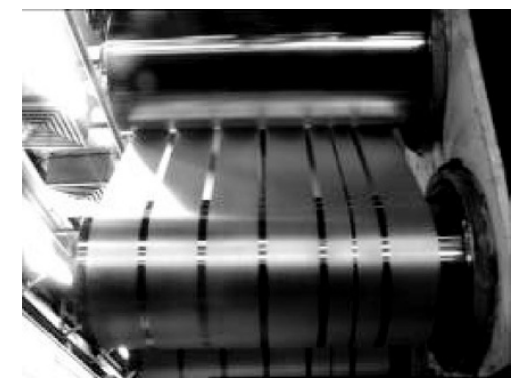

(a)

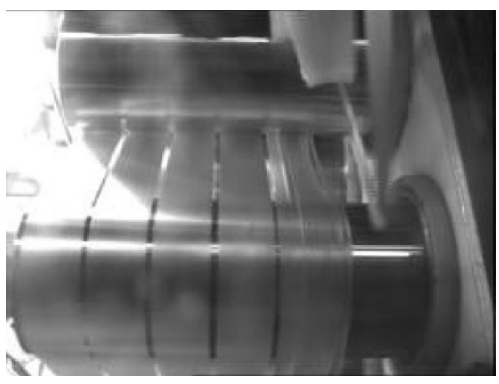

(c)

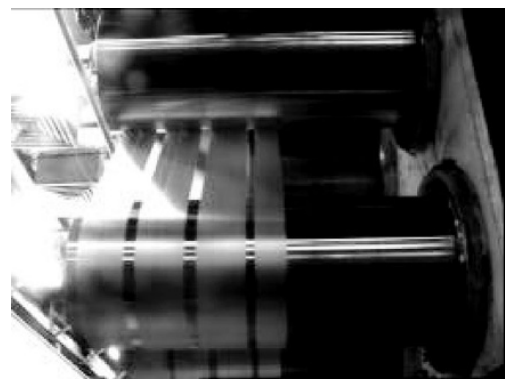

(b)

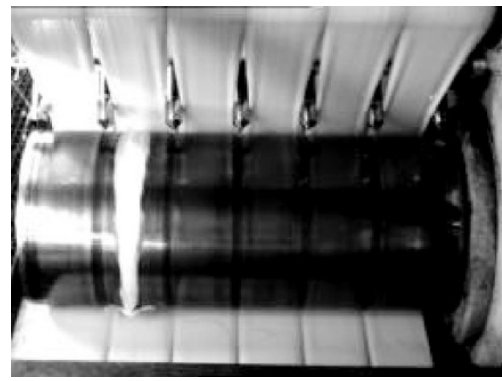

(d)

FIGURE 8: Examples of defects detected by the INFIBRA system: (a) tape gap in the fifth tape; (b) tape width in the fourth tape and missing fifth and sixth tapes; (c) spacing between fourth and fifth tape, fifth tape width, sixth tape missing and wrap presence on the upper cylinder; (d) wrap around cylinder.

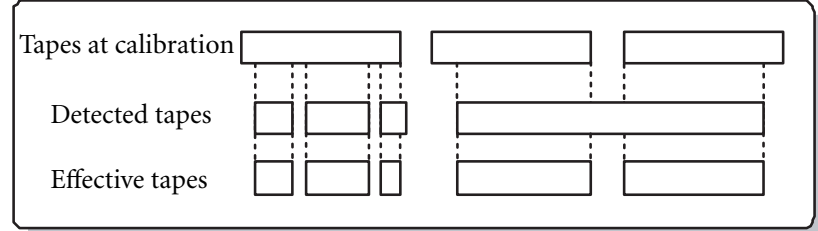

FIgURE 9: Tape presence detection.

detected, the threshold is gradually lowered until a point is reached where all the specified tapes are detected and the resulting threshold, minus a safety margin, is stored for later use.

The tape calibration process also determines the average width of the detected tapes and computes the pixel size (average pixel width in millimeters). The position and width of all tapes are also stored for later detecting the absence of tapes in their expected positions and also for merging fragmented tapes.

The wrap calibration process is similar to that used for the tapes. The wrap inspection windows are often placed over cylinders where oxidation or reflections cause vertical bands, which could be mistaken for wraps. The tape/wrap detection algorithm, when executed within these windows using the optimal threshold, should ignore these bands and return zero wraps. The detection algorithm is executed iteratively

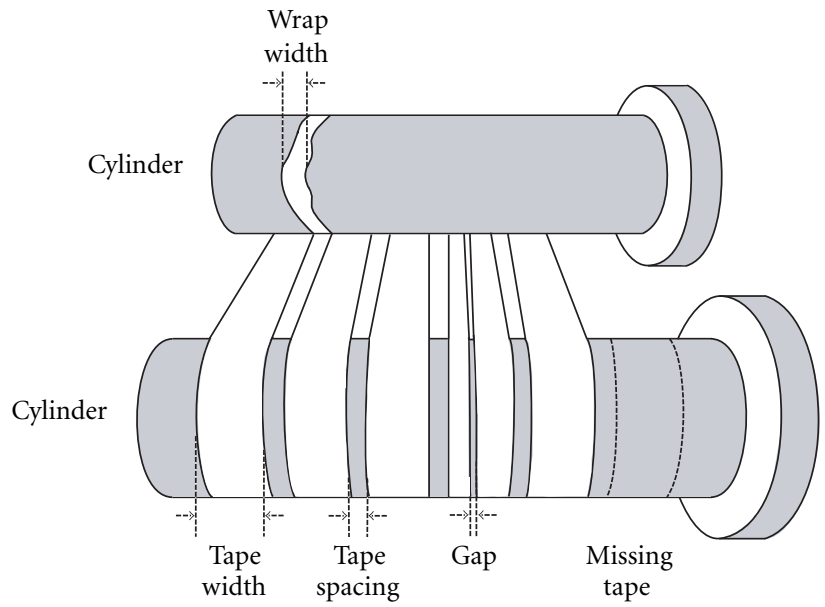

FIgURE 10: Tape and wrap monitoring.

but, contrary to tape calibration, begins with a low threshold and raises it until no wrap is detected. That threshold value (plus a safety margin) is stored as the noise-filtering threshold for the inspection window. The operator, however, must introduce the pixel size manually because no reference wrap could be used (wraps are rather rare and have no fixed width). The pixel size computed in the nearest adjacent tape inspection window is often used as a reference value. 


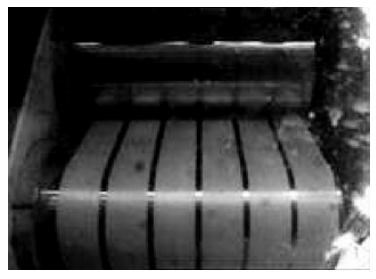

(a)

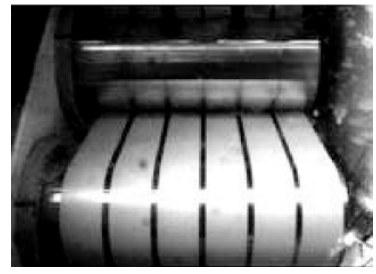

(b)

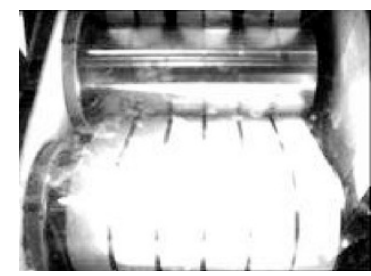

(c)

FIGURE 11: Strong temporal variation of illumination at an inspection station.

\section{RESULTS}

Since the introduction of the INFIBRA system, the client company has been able to eliminate the routine manual visual inspection of acrylic fibre production lines. Instead, a single operator controls the automatic visual inspection from the relative comfort of the control room. The machine vision solution developed is able to monitor the tape width and spacing, and also detect tape gaps and wraps. Any anomaly detected by the system is immediately identified to the operator, who can view the inspection station image via the central control PC and decide what intervention is necessary. INFIBRA can also be configured to send a pager message to the operator when a problem is detected.

A log file reports the events selected by the production line manager. This log file is being integrated into the factory quality control statistical processes. The system ran under close observation for a test period with $100 \%$ defect detection rate and minimal false detections. The manufacturer has not, until now, returned any additional quantitative statistical results. Some of the false detection errors occurred in a particular inspection station, which was later found to have extreme illumination variations during the day (Figure 11). The images show the inspection station at three different times of the day. The last image is highly saturated by the strong reflection of direct sunlight from a nearby window. This problem was later solved by the manufacturer using a screen to block direct sunlight.

\section{CONCLUSIONS}

This multidisciplinary $\mathrm{R} \& \mathrm{D}$ project has resulted in an innovative and unique system that demonstrates the integration of diverse technologies, notably machine vision, and their successful application to a real-world quality control problem. The specific problems to be solved and the consequent objectives of the INFIBRA system were discussed. An overview of the system was given and the image processing algorithms developed were presented in detail.

\section{REFERENCES}

[1] R. C. Gonzalez and P. Wintz, Digital Image Processing, AddisonWesley, Reading, Mass, USA, 2nd edition, 1987.
Paulo Heleno graduated in electrical engineering at the Instituto Superior Técnico, Technical University of Lisbon, from 1998 to 2002 he collaborated with the Artificial Vision Group at INETI's Optoelectronics Department. His current research interests include computer vision, image analysis algorithms and computer graphics.

Roger Davies received a B.S. degree in aeronautical engineering from the Imperial College of Science and Technology, London, in 1982; and a Master's degree in industrial design engineering from the Royal College of Art, London, in 1984. He subsequently worked as a Research Fellow at the RCA investigating computer solid modelling as a tool for industrial design, before joining the

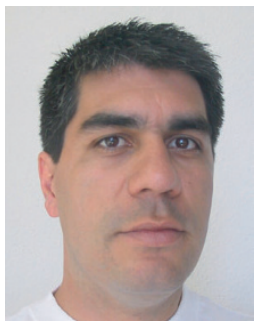
Structural Dynamics Research Corporation (SDRC) in 1987 as a Design Consultant. At SDRC he worked in the areas of computer-aided design, visualisation, and analysis on various projects ranging from a pocket telephone to the interior of a commercial jet aircraft. Since January 1990, he has been carrying out R\&D activities at INETI in Lisbon, Portugal, where he is currently the coordinator of the Vision and Software Engineering Group within the Optoelectronics Department. He has published several papers on machine vision applications and holds three patents for industrial inspection systems based on machine vision.

Bento A. Brázio Correia was born in 1964, graduated in physics from the University of Lisbon in 1987 and received the Ph.D. in physics engineering from Instituto Superior Técnico in 1998. He is a Research Officer at the Department of Optoelectronics of INETI (the Portuguese National Institute of Engineering and Industrial Technology). He has been working since 1986 in the fields of computer vision, digital image process-

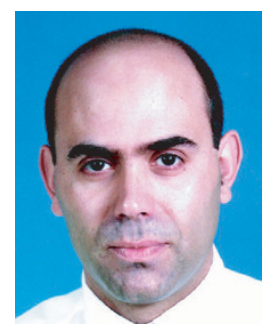
ing, image based metrology, infrared imaging, and automatic target recognition. He was the project manager for the Portuguese participation in the EUCLID RTP 8.2 program for new infrared search and track technologies; and since 2001 he is involved in two ESA projects in the field of optical autonomous navigation for interplanetary missions and for planetary approach and landing. He has published more than 30 papers and was awarded the Youth Prize for his work on New Technologies and Innovation by IEFP in 1987, 
the Descartes Prize by the Instituto de Informática in 1991, the Innovation Prize by ANIMEE in 1995, and the Exemplary Performance Presentation Award by SPIE in 1998.

João Dinis received a degree in engineering physics from the Faculdade de Ciências da Universidade de Lisboa in 1993. Since 1993, he has been carrying out R\&D activities at INETI in Lisbon, Portugal, within the Vision and Software Engineering Group. He has developed both machine visions systems, for industrial applications, and image processing algorithms, for the analysis of infrared images.

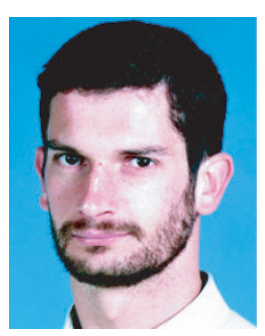

